\title{
Precision Determination of Electron Scattering Angle by Differential Nuclear Recoil Energy Method
}

\author{
N. Liyanage ${ }^{\mathrm{a}}$, K. Saenboonruanga, ${ }^{\mathrm{a}, \mathrm{b}, *}$ \\ ${ }^{a}$ Physics Department, University of Virginia, Charlottesville, VA 22903, USA \\ ${ }^{b}$ Department of Applied Radiation and Isotopes, Faculty of Science, Kasetsart University, \\ Chatuchak, Bangkok, 10900, Thailand
}

\begin{abstract}
The accurate determination of the scattered electron angle is crucial to electron scattering experiments, both with open-geometry large-acceptance spectrometers and ones with dipole-type magnetic spectrometers for electron detection. In particular, for small central-angle experiments using dipole-type magnetic spectrometers, in which surveys are used to measure the spectrometer angle with respect to the primary electron beam, the importance of the scattering angle determination is emphasized. However, given the complexities of large experiments and spectrometers, the accuracy of such surveys is limited and insufficient to meet demands of some experiments. In this article, we present a new technique for determination of the electron scattering angle based on an accurate measurement of the primary beam energy and the principle of differential nuclear recoil. This technique was used to determine the scattering angle for several experiments carried out at the Experimental Hall A, Jefferson Lab. Results have shown that the new technique greatly improved the accuracy of the angle determination compared to surveys.
\end{abstract}

Keywords: High Resolution Spectrometer Angle, Nuclear recoil technique, Jefferson Lab, Hall A, PREX, HAPPEX-III

\footnotetext{
* Corresponding author

Email address: fscikssa@ku.ac.th (K. Saenboonruang )
}

Preprint submitted to Nuclear Instruments 63 Methods in Physics Research AAugust 27, 2015

(C) 2015. This manuscript version is made available under the Elsevier user license http://www.elsevier.com/open-access/userlicense/1.0/ 


\section{Introduction} $5 \theta$ by:

The accurate determination of the scattered electron angle $(\theta)$ is crucial to most of electron scattering experiments, which rely heavily on the accuracy of the four-momentum transfer squared $\left(Q^{2}\right)$ measurement. Since $Q^{2}$ is related to

$$
Q^{2}=2 E E^{\prime}(1-\cos (\theta))
$$

the $\theta$ measurement plays a key role in elastic scattering experiments where both cross section $(\sigma)$ and the form factors strongly depend on $Q^{2}$. The two other factors of $Q^{2}$ measurement are the energies of the incoming and scattered electrons. Until about three to four decades ago, the uncertainties in $Q^{2}$ were dominated by the uncertainties in these energy measurements and not by the uncertainties in the scattering angle determination. In fact, the differential nuclear recoil method was used in some cases to extract the scattered electron energy given the incoming electron energy and the scattering angle [1]. Because of the improvement in present electron scattering experiments, the scattered and incoming electron energies are determined with high precision using high resolution magnetic spectrometers and many independent beam energy measurement techniques. As a result, the accuracy of the scattering angle measurement becomes the limiting factor in the accuracy of $Q^{2}$. This is especially the case for forward-angle electron scattering experiments, where the scattering angles are small (up to about $10^{\circ}$ ).

The scattering angle $(\theta)$ of an electron is defined as the angle between the direction of the scattered electron and the direction of the primary electron beam. In the case of dipole-based magnetic spectrometers, the scattering angle is calculated using a combination of

- the angle of the spectrometer axis with respect to the ideal beam line $\left(\theta_{0}\right)$, and

- the horizontal and vertical angles of the scattered electron $\left(\phi_{t g}\right.$ and $\left.\theta_{t g}\right)$ with respect to the spectrometer axis. 
An illustration and a relation between $\theta, \theta_{t g}, \phi_{t g}$ are shown in Fig. 1 and Eq. 2

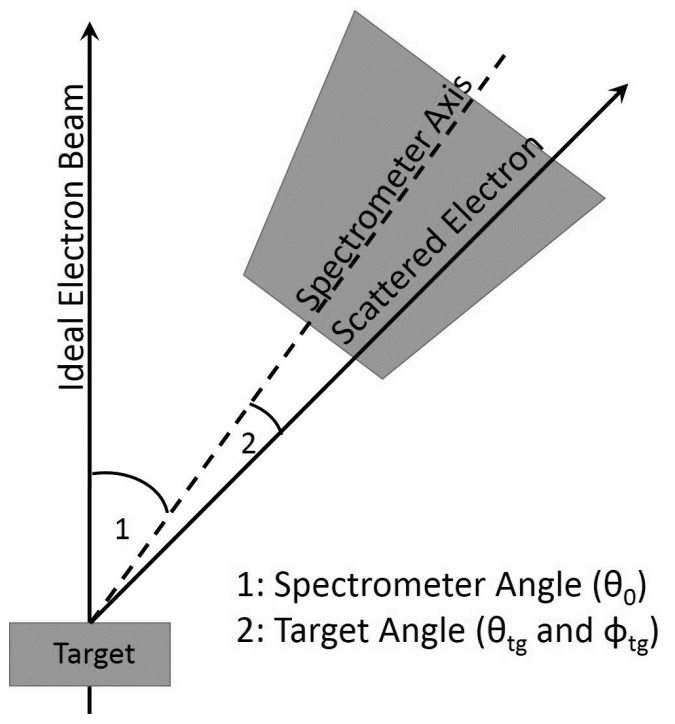

Figure 1: The figure shows the conventional definitions of the spectrometer central angle $\left(\theta_{0}\right)$ and the target angles $\left(\theta_{t g}\right.$ and $\left.\phi_{t g}\right)$. The scattering angle for each event is calculated using $\theta_{0}, \theta_{t g}$, and $\phi_{t g}$. The figure is shown as viewed from above.

30

$$
\theta=\arccos \left(\frac{\cos \left(\theta_{0}\right)-\phi_{t g} \sin \left(\theta_{0}\right)}{\sqrt{1+\theta_{t g}^{2}+\phi_{t g}^{2}}}\right)
$$

Generally, surveys are used to determine $\theta_{0}$ by measuring the angle between two imaginary lines: the first line along the ideal spectrometer axis and the second line along the ideal beam direction. This angle measurement was performed using special equipment from the Stanford Industrial Measurement System (SIMS) 2, 3] and later was updated to an advanced system of optical, laser, and touch probe [4 [5] to locate positions of the target center and the ideal spectrometer center and generally has an angular uncertainty of approximately $0.5 \mathrm{mrad}\left(0.03^{\circ}\right)$. However, multiple corrections required to relate the ideal spectrometer angle to the actual spectrometer angle including target position, and the position of the sieve-slit center with respect to the nominal central ray, increase the uncertainty of $\theta_{0}$ to close to $1.0 \mathrm{mrad}\left(0.06^{\circ}\right)$. Such an uncertainty 
is unacceptably high for some forward angle experiments where the scattering angle itself is in the range of $10^{\circ}$ or smaller. The obvious example is PREX [6], where the central angle was very small ( $\sim 87 \mathrm{mrad})$. The angle uncertainty of $1 \mathrm{mrad}$ would lead to uncertainties of up to $<1 \%$ in angle determination and up to $<2 \%$ in $Q^{2}$ determination. These values of uncertainties were twice larger than the experiment requirements.

In this article, we present a novel technique to determine the electron scattering angle. This technique called the nuclear recoil technique is based on the idea of differential nuclear recoil for elastic electron scattering off target nuclei. This technique has already been used to determine the scattering angle for many experiments carried out in Experimental Hall A at Jefferson lab including HAPPEX-III [7], PVDIS 8] [9], PREX, and G2p [10]. These experiments used the Jefferson Lab Hall A High Resolution Spectrometer (HRS) pair to detect the scattered electrons at forward angles with high precision.

The Jefferson Lab Hall A High Resolution Spectrometers (HRS) are a pair of identical vertically bending spectrometers called L-HRS and R-HRS, each with a combination of three quadrupoles (Q1, Q2, and Q3) and a dipole magnet to focus and to bend the scattered electrons. As described in [11], the $\theta_{t g}$ and $\phi_{t g}$ angle calibration for each HRS is done using a sieve-slit collimator mounted at the entrance of the spectrometer and data from electrons elastically scattered off a set of thin foil targets. In this case, the calibrated angles are referenced with respect to the line that connects the central sieve slit hole to the center of the target foil. However, in order to combine the calibrated $\theta_{t g}$ and $\phi_{t g}$ with $\theta_{0}$ measured using surveys, corrections for the following offsets need to be applied:

- the vertical and horizontal offsets of central sieve-slit hole with respect to the spectrometer axis,

- the offset (or the mis-pointing) of the spectrometer axis with respect to the hall center,

- the offset of the central target foil with respect to the hall center. 
Each of these offsets needs to be measured when performing surveys, which carries an uncertainty of approximately $0.5 \mathrm{~mm}$. These correction terms consequently increase the overall uncertainty of the scattering angle measurement. Furthermore, the required surveys cost many hours of downtime for the experiment following each spectrometer angle change. On the other hand, the nuclear recoil technique does not require any of the corrections mentioned above, and thus, can significantly improve the accuracy of the measurement.

\section{Nuclear Recoil Technique by Differential Nuclear Recoil Energy in Electron Scattering}

In order to perform the nuclear recoil technique, the ability to determine scattered electron energy with high accuracy must be achieved. For experiments in Jefferson Lab Experimental Hall A, the scattered electron energy is measured using two identical HRSs. The standard HRS has an absolute energy accuracy in the range of $\sim 3 \times 10^{-4}$ for the energy of $0.3-4.0 \mathrm{GeV}$. However, two of the forward-angle experiments listed above, PREX and G2p, required an additional dipole magnet in front of each HRS to bend scattered electron from $5^{\circ}$ to $12.5^{\circ}$. This modification required a recalibration of both HRS. The recalibration procedures are described in [12]. The final accuracy of the scattered electron energy measurement for PREX was found to be better than $0.1 \%$. Given that the HRS dipole magnetic field has an accuracy at the $10^{-5}$ level, energy differences between energy spectrum peaks could be determined to a few times $10^{-5}$ level of accuracy. This was tested during HAPPEX-III and PREX calibrations by measuring the differences in the energy of elastically scattered electrons off ${ }^{12} \mathrm{C}$ nucleus and energies of scattered electrons that excited ${ }^{12} \mathrm{C}$ to first excited state $\left(\Delta E_{C}\right)$, which is well measured and known to be $4.433 \pm 0.005 \mathrm{MeV}$. The accuracy of measuring $\Delta E_{C}$ was within $40 \mathrm{keV}$ for both HAPPEX-III and PREX, better than the required accuracy of the experiment. 
Consider the equation for an electron scattering off a target of mass $M_{t}$

$$
E^{\prime}=\frac{\left(E-E_{l o s s 1}\right)-E_{x}\left(1+\frac{E_{x}}{2 M_{t}}\right)}{1+\frac{2\left(E-E_{l o s s 1}\right) \sin ^{2}\left(\frac{\theta}{2}\right)}{M_{t}}}-E_{\text {loss } 2}
$$

where, $E, E^{\prime}, \theta, E_{l o s s 1}, E_{l o s s 2}$, and $E_{x}$, are the beam energy, the energy of the scattered electron, the scattering angle, the energy loss occurred before the scattering, the energy loss occurred after the scattering, and the energy required to excite the target nucleus, respectively. In the case of elastic electron scattering, the $E_{x}$ term vanishes and the equation becomes

$$
E^{\prime}=\frac{E-E_{l o s s 1}}{1+\frac{2\left(E-E_{l o s s 1}\right) \sin ^{2}\left(\frac{\theta}{2}\right)}{M_{t}}}-E_{l o s s 2}
$$

For the kinematics of Jefferson Lab forward angle experiments $(E \geq 1 \mathrm{GeV}$ and $\theta \leq 10^{\circ}$ ) and for scattering off thin foil targets, the above equation could be simplified to:

$$
E^{\prime}=\frac{E}{1+\frac{2(E) \sin ^{2}\left(\frac{\theta}{2}\right)}{M_{t}}}-E_{\text {loss }}+\operatorname{correction}\left(M_{t}, \theta, E, E_{\text {loss } 1}\right)
$$

where $E_{l o s s}=E_{l o s s 1}+E_{l o s s 2}$ is the total energy loss in the target assuming that the scattering took place at the halfway point within the target and the $\operatorname{correction}\left(M_{t}, \theta, E, E_{l o s s 1}\right)$ is a negligible correction term.

A nuclear recoil technique makes use of the fact that, since $E$ and $M_{t}$ are well measured and known, one could calculate $\theta$ with high precision by accurately measuring $E^{\prime}$. Moreover, the systematic uncertainties associated with absolute measurements of $E$ and $E^{\prime}$ could be avoided by measuring the difference in $E^{\prime}$ for electrons elastically scattered off different nuclei $\left(\Delta E^{\prime}\right)$ as shown in Eq. 6 .

$$
\begin{aligned}
\Delta E^{\prime} & =E_{1}^{\prime}-E_{2}^{\prime} \\
& =E\left(\frac{1}{1+\frac{2 E \sin ^{2}\left(\frac{\theta}{2}\right)}{M_{1}}}-\frac{1}{1+\frac{2 E \sin ^{2}\left(\frac{\theta}{2}\right)}{M_{2}}}\right)-\Delta E_{\text {loss }}
\end{aligned}
$$

15 where $\Delta E_{\text {loss }}=E_{\text {loss,target } 1}-E_{\text {loss,target } 2}$ is the difference in total energy losses from the two targets used. The highest sensitivity for this measurement is achieved by using the lightest nucleus, $\mathrm{H}$, as one of the nuclei. 
As shown in Eq. 6, significant uncertainties still arise due to the estimate of energy losses occurred in the target, that could severely affect the accuracy of the nuclear recoil technique. To enhance the accuracy, a nuclear recoil technique could be performed by considering the difference in $E^{\prime}$ for electrons elastically scattered off two different nuclear species in the same target. Examples of targets that can be used for the nuclear recoil technique are a watercell target $\left(\mathrm{H}_{2} \mathrm{O}\right)$, a polyethylene foil $\left(\mathrm{C}_{2} \mathrm{H}_{4}\right)_{n} \mathrm{H}_{2}$ or frozen ammonia $\left(\mathrm{NH}_{3}\right)$. In this case, the energy loss terms drop out (except for the negligible correction term). Furthermore, the angle measurement is completely insensitive to the individual values of $E$ or $E^{\prime}$ and only depends on the difference in energies of electrons elastically scattered off the two different nuclear species in the same target. More importantly, this measurement is unaffected by fluctuations in the beam energy and the beam position during a run.

The advantage of using differences in $E^{\prime}$ of two different nuclei in the same target was emphasized by using PREX as an example. For PREX, the spectrometer was set at a nominal central angle of $5^{\circ}$, while the electron beam energy was set to $1063 \pm 1 \mathrm{MeV}$. Although higher beam energies would have increased the sensitivity of the nuclear recoil technique by increasing $\Delta E$, the constraints of the experiment and the accelerator required that the nuclear recoil technique was carried out with the $1063-\mathrm{MeV}$ electron beam. For this kinematic setting, $\Delta E^{\prime}$ of elastic peaks of hydrogen and any other nucleus was less than $4 \mathrm{MeV}$. As a result, in order to meet the $\theta_{0}$ determination requirement, if $\Delta E^{\prime}$ is measured using two different targets, values of energy loss must be known to $1 \%$ or $40 \mathrm{keV}$, which is difficult to achieve. However, the concern is sufficiently suppressed by considering electrons scattered off two different nuclei in the same target such as watercell or frozen ammonia.

For example, in the case of a watercell target, the energy difference of elec- 


$$
\begin{aligned}
\Delta E^{\prime} & =E_{O}^{\prime}-E_{H}^{\prime} \\
& =E\left(\frac{1}{1+\frac{2 E \sin ^{2}\left(\frac{\theta}{2}\right)}{M_{O}}}-\frac{1}{1+\frac{2 E \sin ^{2}\left(\frac{\theta}{2}\right)}{M_{H}}}\right)+\operatorname{correction}\left(M_{O}, M_{H}, \theta, E, E_{\text {loss } 1}\right)
\end{aligned}
$$

trons elastically scattered off oxygen and hydrogen nuclei is given by:

For the PREX kinematics given above, the correction term was approximately $1.87 \mathrm{keV}(0.05 \%)$ compared to the first term of $\sim 4 \mathrm{MeV}$. The omission of the correction term contributed $\sim 0.03 \%$ to the total uncertainty of the $\theta$ measurement, and thus negligible. This also holds for the ammonia target.

\subsection{Spectrometer Angle ( $\left.\theta_{0}\right)$ Measurement}

To perform the nuclear recoil technique, the watercell target was used in PREX and HAPPEX-III. The sieve slit plate was inserted at the entrance of the spectrometers to provide a reference track and to minimize the dependence on the angular calibration. However, a measurement without the insertion of the sieve slit plate can still be used for a consistency check. In order to determine $\theta_{0}$, only events going through the central sieve slit hole were selected to calculate their corresponding energies.

Figure 2 show the histograms of the energy of scattered electrons and the energy differences between the elastic peaks from hydrogen and oxygen and the first excited state of oxygen, scattered from a watercell in HAPPEX-III. The nuclear recoil technique was done with several spectrometer central momentum settings $\left(P_{0}\right)$ so that their peaks were located in different areas across the HRS focal plane to increase the accuracy. Each of the HRS settings provided an independent measurement of the separation between the oxygen and hydrogen peaks. To accurately measure the energy differences, the shifts in locations of peaks due to the radiative tails must be considered as well. The most important was a possible shift of the hydrogen peak on top of the radiative tail of the oxygen peak for PREX. However, this effect was negligible since the radiative tail of the oxygen peak was relatively flat and the shift in the hydrogen peak was less than $0.1 \%$. 


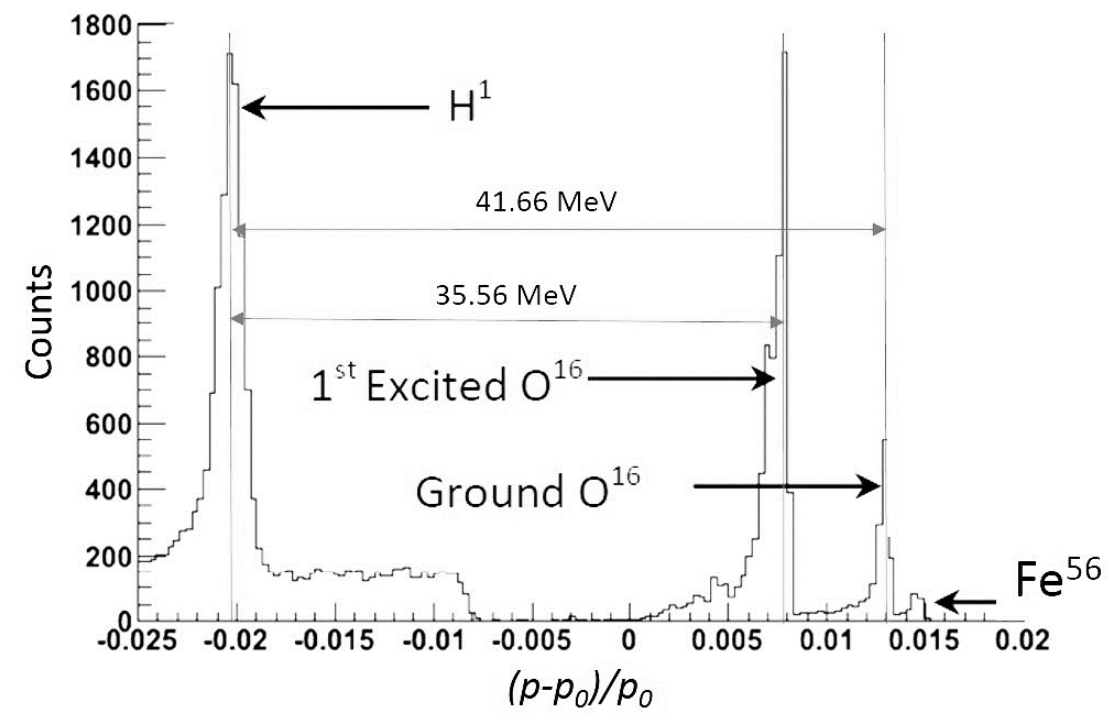

Figure 2: The figure shows the energy distribution of electrons scattered off watercell target in HAPPEX-III. The energy differences between the elastic scattering of hydrogen, oxygen, and the first excited state of oxygen are shown. $p$ is the momentum of scattered electrons and $p_{0}$ is the central momentum setting of the spectrometer.

Energies of electrons scattered off the watercell measured from different $P_{0}$ settings and the energy differences for HAPPEX-III and PREX are presented in Table 1 and 2 respectively. 


\begin{tabular}{|l|c|c|c|c|c|c|}
\hline Spectrometer & $P_{0}(\mathrm{GeV})$ & $\begin{array}{c}E_{\text {O,ground }}^{\prime}(\mathrm{GeV}) \\
(1)\end{array}$ & $\begin{array}{c}E_{O, 1 s t}^{\prime}(\mathrm{GeV}) \\
(2)\end{array}$ & $\begin{array}{c}E_{H}^{\prime}(\mathrm{GeV}) \\
(3)\end{array}$ & $\begin{array}{c}(\mathrm{GeV}) \\
(1)-(3)\end{array}$ & $\begin{array}{c}(\mathrm{GeV}) \\
(2)-(3)\end{array}$ \\
\hline R-HRS & 1.22003 & 1.19345 & 1.18733 & N/A & N/A & N/A \\
\hline R-HRS & 1.20197 & N/A & 1.18743 & 1.15433 & N/A & 0.03309 \\
\hline R-HRS & 1.17799 & 1.19329 & 1.18720 & 1.15411 & 0.03918 & 0.03310 \\
\hline R-HRS & 1.16601 & 1.19325 & 1.18719 & 1.15411 & 0.03914 & 0.03309 \\
\hline L-HRS & 1.22002 & 1.19338 & 1.18731 & N/A & N/A & N/A \\
\hline L-HRS & 1.20201 & 1.19336 & 1.18730 & N/A & N/A & N/A \\
\hline L-HRS & 1.17798 & 1.19321 & 1.18709 & 1.15159 & 0.04162 & 0.03550 \\
\hline L-HRS & 1.16600 & 1.19321 & 1.18711 & 1.15155 & 0.04166 & 0.03556 \\
\hline
\end{tabular}

Table 1: Values of the electron energy scattered and the energy differences from the watercell in HAPPEX-III. "N/A" denotes that the peak is too small to be measured. The uncertainty of the measurement is $\sim 0.1 \%$.

\begin{tabular}{|l|c|c|c|c|}
\hline Spectrometer & $P_{0}(\mathrm{GeV})$ & $\begin{array}{c}E_{\text {O,ground }}^{\prime}(\mathrm{GeV}) \\
(1)\end{array}$ & $\begin{array}{c}E_{H}^{\prime}(\mathrm{GeV}) \\
(2)\end{array}$ & $\begin{array}{c}(\mathrm{GeV}) \\
(1)-(2)\end{array}$ \\
\hline R-HRS & 1.063 & 1.06115 & 1.05706 & 0.00409 \\
\hline R-HRS & 1.058 & 1.06129 & 1.05713 & 0.00416 \\
\hline R-HRS & 1.047 & 1.06121 & 1.05710 & 0.00411 \\
\hline L-HRS & 1.063 & 1.06120 & 1.05696 & 0.00424 \\
\hline L-HRS & 1.058 & 1.06115 & 1.05694 & 0.00421 \\
\hline L-HRS & 1.047 & 1.06117 & 1.05690 & 0.00427 \\
\hline
\end{tabular}

Table 2: Values of the electron energy scattered and the energy differences from the watercell in PREX. The uncertainty of the measurement is $\sim 0.1 \%$.

$\Delta E_{\text {measured }}^{\prime}$ and the expected scattered electron energies $\left(\Delta E^{\prime}\right)$ calculated from Eq. 7, were used to calculate $\theta_{0}$ using the least-squares method, which minimized the sums of $\left(\Delta E^{\prime}-\Delta E_{\text {measured }}^{\prime}\right)^{2}$ from all $P_{0}$ settings, treating $\theta$ as the parameter to be optimized. The value of $\theta$ obtained from the optimization would be the angle between the direction of the ideal electron beam and a line from the target center to the center of the central sieve slit hole. To translate this calculated value of $\theta$ to the conventional definition of $\theta_{0}$ explained in Section 1 . the measurement of the misalignment of the center of the central sieve slit hole needed to be measured relative to the center of the spectrometer. However, to avoid any additional uncertainty from measuring the misalignment, we can adequately redefine the spectrometer angle, $\theta_{0}$, to this calculated $\theta$ and hence, 
redefine target angles and other variables to be measured with respect to this new defined spectrometer angle as shown in Fig. 3 .

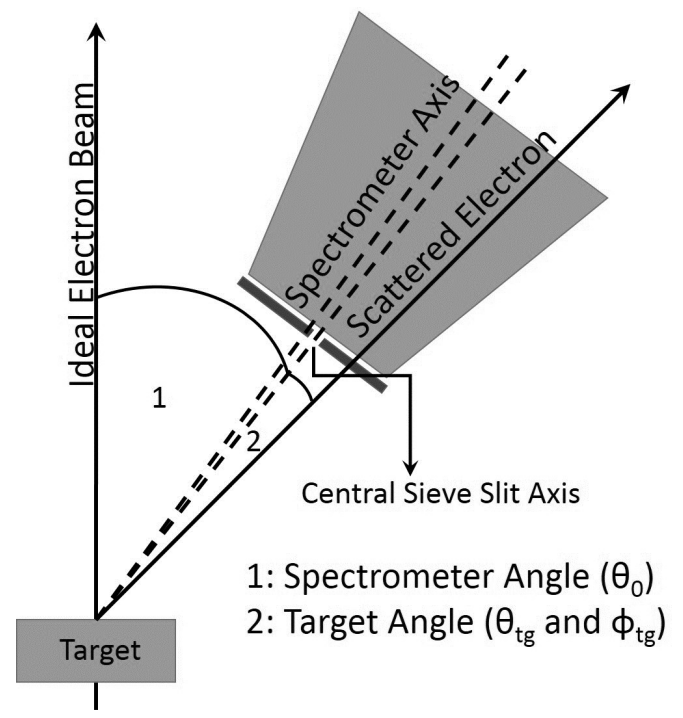

Figure 3: The figure illustrates the modified definitions of the spectrometer angle $\left(\theta_{0}\right)$ and target angle $\left(\theta_{t g}\right.$ and $\left.\phi_{t g}\right) . \theta_{0}$ is now defined as an angle between the direction of the ideal electron beam and a line from the target center to the center of the central sieve slit hole.

\section{Results}

Values of spectrometer angles $\left(\theta_{0}\right)$ and their respective uncertainties for PREX and HAPPEX-III obtained from performing the nuclear recoil technique and surveys are shown in Table 3 and 4.

\begin{tabular}{|l|c|c|}
\hline Spectrometer & Nuclear recoil technique $\left(^{\circ}\right)$ & Surveys $\left(^{\circ}\right)$ \\
\hline L-HRS & $5.065 \pm 0.020$ & $5.007 \pm 0.046$ \\
\hline R-HRS & $4.933 \pm 0.020$ & $4.910 \pm 0.046$ \\
\hline
\end{tabular}

Table 3: Spectrometer angles, $\theta_{0}$, measured by using a nuclear recoil technique and surveys of PREX. 


\begin{tabular}{|l|c|c|}
\hline Spectrometer & Nuclear recoil technique $\left(^{\circ}\right)$ & Surveys $\left(^{\circ}\right)$ \\
\hline L-HRS & $14.078 \pm 0.020$ & $14.015 \pm 0.046$ \\
\hline R-HRS & $13.688 \pm 0.020$ & $13.825 \pm 0.046$ \\
\hline
\end{tabular}

Table 4: Spectrometer angles, $\theta_{0}$, measured by using the nuclear recoil technique and surveys of HAPPEX-III.

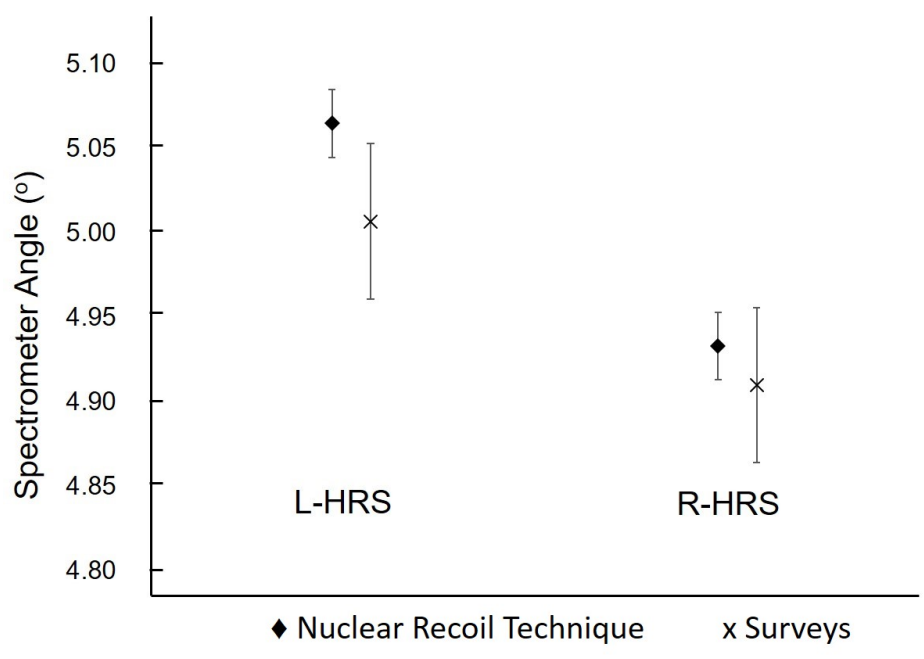

Figure 4: The figure shows comparisons between values and uncertainties of spectrometer angle measured by the nuclear recoil technique and surveys in L-HRS and R-HRS of PREX

As shown in Fig. 5, results from the nuclear recoil technique and surveys for R-HRS disagreed by $\sim 3 \sigma$. This was due to a considerably large misplacement of $\sim 3 \mathrm{~mm}$ between the idea! ${ }^{1}$ and actual target positions [13] and $\sim 1 \mathrm{~mm}$ between the idea $!^{2}$ and actual central sieve slit hole positions [14] in R-HRS. These misplacements required corrections to be applied in surveys in order to report values of $\theta_{0}$, which increased their uncertainties. In the case of L-HRS,

\footnotetext{
${ }^{1}$ The ideal target position is the intersection of the ideal electron beam and the vertical symmetry axis of the target system.

${ }^{2}$ Ideal central sieve slit hole is the intersection of the plane of the sieve slit and the spectrometer axis.
} 


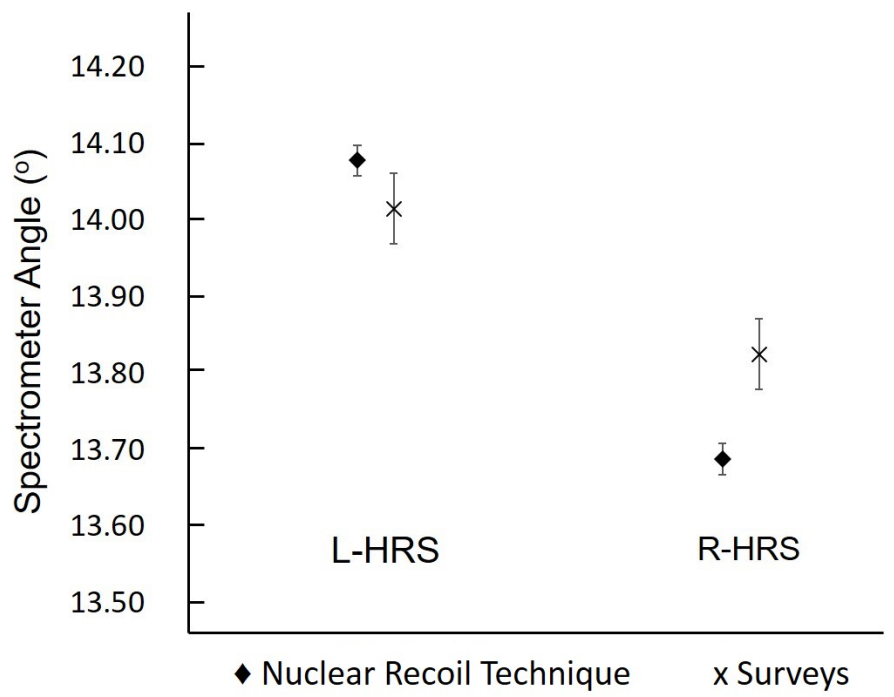

Figure 5: The figure shows comparisons between values and uncertainties of spectrometer angle measured by the nuclear recoil technique and surveys in L-HRS and R-HRS of HAPPEX-III.

the misplacements were less than $1 \mathrm{~mm}$, hence, values from the nuclear recoil technique and surveys agreed within one $\sigma$.

Uncertainties from performing the nuclear recoil technique were $0.4 \%$ and $0.1 \%$, while surveys gave $\sim 1 \%$ and $\sim 0.3 \%$ for PREX and HAPPEX-III respectively. Results have clearly shown that the nuclear recoil technique could double the accuracy of the angle measurement when compared to surveys. The factor that contributed the most to the angle measurement uncertainty was the ability to measure the difference between two different nuclei $(\sim 1 \%)$, while a small contribution $(\sim 0.1 \%)$ was from the uncertainty in electron beam energy measurement.

\section{Conclusions}

The spectrometer angle measurement is crucial for kinematics analyses of electron scattering experiments. In the past, the spectrometer angle was measured mainly by performing surveys. However, with higher demands on the pre- 
cision of the experiments, a new and more accurate method called the pointing measurement, which makes use of the differential nuclear recoil energy of different target nuclei, was developed. This new method was used for the spectrometer angle determination of PREX and HAPPEX-III experiments to improve the scattering angle accuracy by more than a factor of two. The improvement in the scattering angle measurement helped PREX to meet its demanding requirement of $1 \%$ accuracy in $Q^{2}$ measurement by contributing $\sim 0.9 \%$ to the overall systematic uncertainty, which included other uncertainties from primary beam energy and scattered electron energy measurements.

\section{Acknowledgement}

We wish to thank the entire staff of the Jefferson Lab for their efforts to put together the experimental setup, for collaboration during the experiment, and for helping with the analysis after the completion of the experiments. We also thank the PREX and the HAPPEX collaborations for their help with the analysis. Jefferson Science Associates, LLC, operates Jefferson Lab for the U.S. DOE under U.S. DOE contract DE-AC05-060R23177.

\section{References}

[1] E. Offermann, et al., Energy dependence of the form factor for elastic electron scattering from ${ }^{12}$ C, Phys. Rev. C 44 (1991) 1096.

[2] B. Bell, Sims: The slac industrial measurement system, in: Proceedings of the 1st International Workshop on Accelerator Alignment (IWAA 1989), Stanford, California, 1989, p. 162.

[3] C. Curtis, J. Dahlberg, W. Oren, K. Tremblay, Techniques used in the alignment of tjnaf's accelerators and experimental halls, in: Proceedings of the 5st International Workshop on Accelerator Alignment (IWAA 1997), Argonne, Illinois, 1997, p. 038. 
[4] Bigbite spectrometer (September 2008). URL https://hallaweb.jlab.org/wiki/index.php/BigBite_ Spectrometer

[5] M. Battaglieri, et al., The heavy photon search test detector, Nuclear Instruments \& Methods in Physics Research A 777 (2015) 91.

[6] S. Abrahamyan, et al., Measurement of the neutron radius of ${ }^{208} \mathrm{pb}$ through parity violation in electron scattering, Phys. Rev. Lett. 108 (2012) 112502.

[7] Z. Ahmed, et al., New precision limit on the strange vector form factors of the proton, Phys. Rev. Lett. 108 (2012) 102001.

[8] D. Wang, et al., Measurements of parity-violating asymmetries in electrondeuteron scattering in the nucleon resonance region, Phys. Rev. Lett. 111 (2013) 082501.

[9] D. Wang, et al., Measurement of parity violation in electron-quark scattering, Nature 506 (2014) 67.

[10] A. Camsonne, et al., A measurement of $g_{p}^{2}$ and the longitudinal-transverse spin polarizability, Proposal to Jefferson Lab PAC-33 (Jan 2008).

[11] J. Alcorn, et al., Basic instrumentation for hall a at jefferson lab, Nuclear Instruments \& Methods in Physics Research A 522 (2004) 294.

[12] K. Saenboonruang, Measurement of the neutron radius of ${ }^{208} \mathrm{pb}$ through parity violation in electron scattering, Ph.D. thesis, University of Virginia (May 2013).

[13] K. Tremblay, Hall a pointings e081709a, h081809a \& superharps, bcms etc. (August 2009).

URL https://www.jlab.org/accel/survalign/documents/dthalla/ A1241.pdf 
262 [14] J. Dahlberg, Hall a collimator/sieve calibration (August 2009).

263 URL https://www.jlab.org/accel/survalign/documents/dthalla/

$264 \quad$ A1239.pdf 